TITLE:

\title{
Microscopic image restoration based on tensor factorization of rotated patches
}

$\operatorname{AUTHOR}(\mathrm{S}):$

Kouno, Masayuki; Nakae, Ken; Oba, Shigeyuki; Ishii, Shin

\section{CITATION:}

Kouno, Masayuki ... [et al]. Microscopic image restoration based on tensor factorization of rotated patches. Artificial Life and Robotics 2013, 17(3-4): 417-425

ISSUE DATE:

2013-02

URL:

http://hdl.handle.net/2433/172228

\section{RIGHT:}

The final publication is available at www.springerlink.com; この論文は 出版社版でありません。引用の際には出版社版をご確認ご利用くださ $\omega_{\circ}$; This is not the published version. Please cite only the published version. 


\title{
Microscopic image restoration based on tensor
}

\section{factorization of rotated patches}

\author{
Masayuki Kouno $^{1}$, Ken Nakae ${ }^{1}$, Shigeyuki Oba ${ }^{1,2}$, and Shin Ishii ${ }^{1}$ \\ ${ }^{1}$ Graduate School of Informatics, Kyoto University, Gokasho, Uji, Kyoto 611-0011, Japan \\ ${ }^{2}$ PRESTO, Japan Science and Technology Agency \\ Tel: +81-774-38-3938, Fax: +81-774-38-3941 \\ E-mail: kouno-m@sys.i.kyoto-u.ac.jp
}


Abstract- In microscopic image processing for analyzing biological objects, structural characters of objects such as symmetry and orientation can be used as a prior knowledge to improve the results. In this study, we incorporated filamentous local structures of neurons into a statistical model of image patches and then devised an image processing method based on tensor factorization with image patch rotation. Tensor factorization enabled us to incorporate correlation structure between neighboring pixels, and patch rotation helped us obtain image bases that well reproduce filamentous structures of neurons. We applied the proposed model to a microscopic image and found significant improvement in image restoration performance over existing methods, even with smaller number of bases.

Keywords- Image processing, Microscopic image, Tensor factorization, Local feature extraction 


\section{INTRODUCTION}

Image patch factorization is a standard way to extract local structural features in image processing

[1]. A rectangular image patch is cut out from a target image and regarded as a random sample that is generated from a linear sum of base image patches. The set of base image patches is often called a dictionary. If we have a good dictionary, each image patch is well approximated by a factor vector of low dimensionality corresponding to the number of bases, which leads to image compression, efficient image recognition, image de-noising, and image restoration. In the factorization, we estimate the dictionary, a good set of base image patches, in an adaptive manner to fit to the given set of sample patches. In their pioneering work, Olshausen and Field estimated a set of basis image patches from natural images by a maximum likelihood estimation, and found that the best dictionary resembled the set of two-dimensional wavelets [2]. The adaptive learning of dictionary is used for noise reduction by regression [1][3][4], in various application fields, such as natural image, text, and face [5].

In analyses of biological objects with microscopic image processing, local structural characters of the objects can provide a set of prior knowledge to improve the result. For example, neurons consist of long and narrow fibers, i.e., dendrites and axons, spreading from main cell bodies, i.e., soma, to an arbitrary extent of three-dimensional space. Thus, extraction of flexible rotational coordinates along the fiber-like neurite can improve the analyses of microscopic images of neuronal networks.

In order to incorporate prior knowledge of target objects in analysis of microscopic images we introduce the following two strategies: (a) to obtain direction of foreground objects by arranging rotational image patches; and, (b) to handle the set of image patches as a three-way tensor (patch 
height $\times$ patch width $\times \sharp$ patches $)$ rather than a matrix ( $\sharp$ patch pixels $\times \sharp$ patches $)$.

In strategy (a), image patches are rotated to suit a pre-determined template image that has been prepared to represent a part of neural fiber aligned to a certain direction. When this rotation step is exhibited before basis learning step, the obtained set of bases are expected to reflect a variety of fiber-like local structure of neurons, being different from bases of natural image patches which generally look like two-dimensional Fourier bases [2]. In strategy (b), we regard each rectangular image patch as a matrix rather than a vector with the same number of elements and apply a technique of tensor factorization for base learning. Brightness of pixels in an image patch has correlation structure reflecting two-dimensional spatial extensions. A conventional matrix factorization is designed to extract statistical pixel-wise correlation. A tensor factorization can, in addition, incorporate the intrinsic two-dimensional spatial extension structure that the matrix factorization misses. Kim et al. [6] proposed a general purpose image restoration based on tensor factorization. They, however, did not consider symmetric local structure in image patches which we are in particular interested in.

We apply our proposed method to microscopic neuron image processing, and show a significant improvement in the noise reduction.

\section{NOTATIONS FOR TENSOR ANALYSIS}

In this section, we introduce notations necessary to describe the idea of three-way tensor factorization. 


\subsection{TENSOR \& ITS MATRICIZATION}

Let $\underline{\mathbf{Y}} \in \mathbb{R}^{I_{1} \times I_{2} \times I_{3}}$ be a three-way tensor whose real-valued element $y_{i_{1} i_{2} i_{3}} \in \mathbb{R}$ is indexed by $i_{1} \in I_{1}, i_{2} \in I_{2}, i_{3} \in I_{3}$. Each of $I_{1}, I_{2}, I_{3} \in \mathbb{N}$ denotes the largest number of the corresponding index. Let $\|\underline{Y}\|_{\mathrm{F}}^{2}$ denote a squared Frobenius norm, namely, a sum of square of all elements of tensor $\underline{\mathbf{Y}}$.

We sometimes rearrange a multi-way tensor to a matrix form, which is called matricization of the tensor. Mode-3 matricization of a tensor $\underline{\mathbf{Y}}$ is denoted as $\mathbf{Y}_{(3)} \in \mathbb{R}^{I_{3} \times M_{3}}$, where $M_{3}=I_{1} I_{2}$ and the element $\left(i_{1}, i_{2}, i_{3}\right)$ of tensor $\underline{\mathbf{Y}}$ is in the element $\left(i_{3}, i_{1}+\left(i_{2}-1\right) I_{1}\right)$ of matrix $\mathbf{Y}_{(3)}$. Mode-1 and -2 matricizations are similarly defined as to make element $\left(i_{1}, i_{2}+\left(i_{3}-1\right) I_{2}\right)$ of matrix $\mathbf{Y}_{(1)}$ and element $\left(i_{2}, i_{1}+\left(i_{3}-1\right) I_{1}\right)$ of matrix $\mathbf{Y}_{(2)}$ correspond to element $\left(i_{1}, i_{2}, i_{3}\right)$ of tensor $\underline{\mathbf{Y}}$, respectively.

\subsection{TENSOR-MATRIX PRODUCT}

Mode-3 product of a tensor $\underline{\mathbf{G}} \in \mathbb{R}^{J_{1} \times J_{2} \times J_{3}}$ and a matrix $\mathbf{A}^{(3)} \in \mathbb{R}^{I_{3} \times J_{3}}, \underline{\mathbf{Y}}=\underline{\mathbf{G}} \times{ }_{3} \mathbf{A}^{(3)}$, becomes a tensor $\underline{\mathbf{Y}} \in \mathbb{R}^{J_{1} \times J_{2} \times I_{3}}$ whose elements are given by $y_{i_{1} i_{2} i_{3}}=\sum_{j_{3}=1}^{J_{3}} g_{i_{1} i_{2} j_{3}} a_{i_{3} j_{3}}$.

Multiplication of a tensor over all modes other than mode-3 is denoted as

$$
\underline{\mathbf{G}} \times{ }_{-3}\{\mathbf{A}\}=\underline{\mathbf{G}} \times{ }_{1} \mathbf{A}^{(1)} \times{ }_{2} \mathbf{A}^{(2)}
$$

and the resulting tensor has dimensionality $I_{1} \times I_{2} \times J_{3}$.

Mode-1 and -2 products of tensor-matrix are similarly defined. 


\section{METHODS}

In this section, we present our new image restoration method based on tensor factorization and rotational patches.

\subsection{IMAGE PATCH AND ITS FACTORIZATION}

An image patch $\mathcal{Y} \in \mathbb{R}^{m \times m}$ is an $m \times m$ part of the objective image of arbitrary size. An $m \times m$ image patch can also be represented by a vector $\mathbf{y} \in \mathbb{R}^{m^{2}}$ of the same number of elements. In our image restoration method, an image patch vector is approximated by a linear combination of bases,

$$
\begin{aligned}
\mathbf{y} & \simeq \sum_{k=1}^{K} w_{k} \mathbf{x}_{k} \\
& =\mathbf{X} \mathbf{w}
\end{aligned}
$$

where $\mathbf{x}_{k} \in \mathbb{R}^{m^{2}}$ is the $k$-th basis vector and $w_{k} \in \mathbb{R}$ is the corresponding weight coefficient. In image patch factorization or basis learning, the set of bases $\mathbf{X}=\left[\mathbf{x}_{1}, \mathbf{x}_{2}, \cdots, \mathbf{x}_{K}\right]$ is obtained from $N$ samples of image patches $\mathbf{Y} \in \mathbb{R}^{m^{2} \times N}$. And, in basis application, the weight coefficient $\mathbf{w}=$ $\left(w_{1}, w_{2}, \cdots, w_{K}\right)$ is determined for a test image patch $\mathbf{y} \in \mathbb{R}^{m^{2}}$ by solving a minimization problem with fixing the set of bases $\mathbf{X}$ :

$$
\hat{\mathbf{w}}=\underset{\mathbf{w}}{\arg \min }\|\mathbf{y}-\mathbf{X w}\|_{\mathrm{F}}^{2}
$$

Then the image patch is reconstructed by $\hat{\mathbf{y}}=\mathbf{X} \hat{\mathbf{w}}$.

In order to achieve a good set of image bases, we utilized tensor factorization rather than matrix factorization. 


\subsection{IMAGE RESTORATION INCORPORATING PATCH ROTATION}

Our image restoration method is a modification of the tensor factorization method proposed by

Kim et al. [6]. Their method consisted of two steps; a basis learning step and a basis application step.

We added a patch rotation process to both of these steps in order to incorporate symmetric structural features of image patch bases.

In the basis learning step, an image patch tensor consisting of $p$ samples of $m \times m$ rectangular image patches is constructed from a reference image. Note here that we gather image patches from foreground area of the reference image; namely, we omit background area to extract local structure of the foreground objects. In specific, we selected $p$ foreground pixels which are brighter than the pre-determined level, got $p$ samples of foreground patches whose central pixel is one of the selected foreground pixels, and arranged all the sampled patches into an $m \times m \times p$ three-way tensor, which is called a train patch tensor.

We calculate a basis tensor $\underline{\Omega} \in \mathbb{R}^{m \times m \times K}$ as a solution of the minimization problem:

$$
\underline{\boldsymbol{\Omega}}=\underset{(\underline{\boldsymbol{\Omega}}, \mathbf{W})}{\arg \min }\left\|\underline{\mathbf{Y}}-\underline{\boldsymbol{\Omega}} \times{ }_{3} \mathbf{W}\right\|_{\mathrm{F}}^{2},
$$

where $\mathbf{W}$ is a $p \times K$ mixing matrix such to allow the linear sum of $K$ basis patches $\Omega_{k}(k=1, \cdots, K)$ to well approximate the train patch tensor $\underline{\mathbf{Y}}$; that is, the $i$-th patch $\mathcal{Y}_{i}$ is well approximated by a linear sum of the basis patches $\sum_{k=1}^{K} \mathbf{W}(i, k) \boldsymbol{\Omega}_{k}$ for all $i$. The number $K(<p)$ of basis patches should be set appropriately by hand. This minimization problem is solved by three-way tensor factorization and in particular, higher order orthogonal iteration (HOOI) algorithm was used for it, which will be 
explained in detail in section 3.3.

In the basis application process, we restore a noisy image patch $\mathcal{Y}$ to a restored image patch $\hat{\mathcal{Y}}$ by applying the basis tensor $\Omega$ that was obtained in the basis learning step. In this restoration, we assume that an image patch $\mathcal{Y}$ is expressed by linear combination of $K$ basis patches as follows.

$$
\begin{aligned}
\mathcal{Y} & =\underline{\boldsymbol{\Omega}} \times_{3} \mathbf{w}+\mathbf{E} \\
& =\sum_{k=1}^{K} w_{k} \boldsymbol{\Omega}_{k}+\mathbf{E},
\end{aligned}
$$

where $\Omega_{k}$ is an $m \times m$ matrix that represents the $k$-th basis patch image, w is a $K$-dimensional weight vector, and $\mathbf{E}$ is an $m \times m$ residual matrix. We obtain such a weight vector $\hat{\mathbf{w}}^{\top}=\left(\hat{w}_{1}, \cdots, \hat{w}_{K}\right)$ that minimizes the Frobenius norm of the residual matrix $\|\mathbf{E}\|_{\mathrm{F}}^{2}$. Finally, we obtain the restored image patch $\hat{\mathcal{Y}}=\underline{\Omega} \times{ }_{3} \hat{\mathbf{w}}$. And, the entire image is restored by applying the above restoration process for all patches in the image.

In this study, we introduce a patch rotation process in order to incorporate symmetrical structures image. First, we prepared a template image (the leftmost panel in Fig. 1), which was set manually to represent a local neural filamentous structure in the vertical direction. Next, we prepared a set of its rotated image templates with rotation angles, $1,2, \ldots, 180$ degrees (Fig. 1). In the patch rotation process, we fit each sampled patch to the vertical template in the following manner; calculate correlation coefficients between the sampled patch and the rotated templates of 180 rotation angles, determine the best rotation angle that maximizes the correlation, and rotate the sampled patch into the inverse direction with the determined rotation angle. Note that, in order to reduce the total number of rota- 
tion operation, we rotated the template patch 180 times, rather than the sampled patch, in the above procedure.

Fig. 1 shows some examples of image patches before (middle) and after (lower) the patch rotation.

We applied the patch rotation process to both of the basis learning and basis application steps. Before the basis learning step, we aligned all image patches to suit the template image, and gathered the rotated patches to construct a three-way patch tensor for the basis learning. Before the basis application step, we aligned each image patch to suit the template image, and restored the central pixel based on the result obtained by applying the bases.

Owing to the patch rotation, we can omit trivial variations of foreground patch images which can be generated by the rotation of rectangular spatial cutting windows, and consequently, we expect the set of bases reflect variations due to symmetrical structures.

\subsection{HOOI ALGORITHM FOR BASIS LEARNING STEP}

The HOOI algorithm for the three-way tensor factorization in the basis learning step is based on an iterative Alternative Least Squares (ALS) algorithm. In each iteration of ALS, one basis is updated to minimize the residual error while keeping the others fixed, and finally we seek a (locally) optimal solution [7]. An extension of ALS for tensor factorization, HOOI, was first introduced by De Lathauwer, De Moor and Vandewalle [7] and recently extended and implemented by Kolda and Bader in their MATLAB Tensor Toolbox [8]. We used this toolbox for tensor factorization in this study.

The HOOI algorithm factorizes an $m \times m \times p$ patch tensor $\underline{\mathbf{Y}}$ into an $m \times m \times K$ core tensor $\underline{\mathbf{G}}$ 
and three matrices $\mathbf{A}^{(1)}, \mathbf{A}^{(2)}$, and $\mathbf{A}^{(3)}$,

$$
\underline{\mathbf{Y}}=\underline{\mathbf{G}} \times{ }_{1} \mathbf{A}^{(1)} \times{ }_{2} \mathbf{A}^{(2)} \times{ }_{3} \mathbf{A}^{(3)} .
$$

We calculate the basis tensor that is defined in Eq. (4), $\underline{\Omega} \in \mathbb{R}^{m \times m \times K}$, by using the result of HOOI as, $\underline{\boldsymbol{\Omega}}=\underline{\mathbf{G}} \times{ }_{1} \mathbf{A}^{(1)} \times{ }_{2} \mathbf{A}^{(2)}$.

A pseudo-code for the HOOI algorithm is shown in Fig. 6. In Fig. 6, svds $(\mathbf{Z}, k)$ denotes a singular value decomposition procedure that decomposes a matrix $\mathbf{Z}$ into $\mathbf{Z}=\mathbf{U S V}$, where $\mathbf{U}$ and $\mathbf{V}$ are orthonormal bases of rank $k$, and $\mathbf{S}$ is a diagonal matrix of rank $k$. The cost function $\|\underline{\mathbf{Y}}-\underline{\hat{\mathbf{Y}}}\|_{\mathrm{F}}^{2}$ is monitored to be used as a 'stopping criterion' on line 8 in the HOOI algorithm.

\section{RESULTS}

Image restoration performance was compared between tensor factorization and matrix factorization, and between with and without the patch rotation processes. A microscopic image of size $300 \times 300$ pixels was clipped from the original image (size: $634 \times 800$ pixels) of a neuronal network, registered on www. greenspine.ca (Paul De Koninck, Laval University) (Fig. 2, left). To examine the restoration performance, we artificially added white Gaussian noise (variance $=0.03$ ) to the neuronal image (Fig. 2, right). Since we know the original image, we can evaluate the image restoration performance by calculating the root mean squared error (RMSE) of pixel brightness between the original and restored images. Since the performance depends on the number of bases, $K$, the RMSE was calculated with various $K$ values for each method. For the basis learning, we extracted about 17,000 image patches of size $13 \times 13$ pixels from the original image before noise addition. Al- 
though the original image is not available in the actual image restoration application, we here assume the basis learning has been accomplished by using other clear images prior to the application of our image restoration method. Other possible procedure would be to iterate the basis learning step and image restoration by using the same (possibly noisy) images.

Figs. 3(a) and 3(b) show top 14 of patch factor images obtained by matrix factorization with (a) and without (b) patch rotation, respectively, where each patch factor is reshaped from a vector of $\Omega_{k}$ into a square shape of $13 \times 13$ pixels. Patch factor images without the patch rotation are similar to Fourier bases, while those with the patch rotation seem to reflect filamentous structures of neurons. Similarly, Figs. 3(c) and 3(d) show top 14 patch factor images obtained by three-way tensor factorization with (c) and without (d) patch rotation, respectively. At first glance, the patch factor images obtained by the tensor factorization and the matrix factorization were not significantly different.

The image restoration results are shown in Fig. 4. The image restored by the tensor factorization with patch rotation obviously exhibited finer fiber structures than those by other methods. Such good restoration performance of the proposed method can be further seen in the numerical results summarized in Fig. 5. Since the performance was largely dependent on the number of bases, $K$, we here examined various $K$ values. Although the RMSE of each method became the smallest with an appropriate setting of $K$, with the best setting being dependent on the method, the proposed method, the tensor factorization with patch rotation, showed the least RMSE value in every setting. In addition, tensor factorization (TF) showed better results that matrix factorization (MF), even though the former employed a smaller $K$ value than the latter. 
The advantage of patch rotation was obvious especially when the number of bases was small. Table 1 shows the RMSE with the best setting of $K$ for various noise levels added to the original image; our proposed method showed the best restoration performance with a smaller number of bases in all cases.

\section{CONCLUDING REMARKS}

In this study, we proposed an image restoration method based on three-way tensor factorization of rotated image patches, which is suitable for restoration of microscopic images of biological targets such as neuronal networks. In the simulation experiment using a real image of neuronal network, we found significant performance gain in image restoration due to the two important ingredients of our method, tensor factorization and image patch rotation.

Fluorescence microscopy has a dilemma that an increase in the frame rate suffers from larger shot noise, while a decrease leads to missing dynamic characters of the target. Thus, a good image restoration technique is important for improving the data acquisition efficacy. For example, if we can restore low resolution images by utilizing basis images created from other high resolution images, the original low resolution images are restored cheaply in a software manner. Applications to 3D images and movies would be important future extensions, and capturing various finer structures of neuronal networks by further modifying our current method will also be promising. 


\section{ACKNOWLEDGMENT}

This study was supported by Grant-in-Aid for Scientific Research on Innovative Areas: “ Mesoscopic neurocircuity: towards understanding of the functional and structural basis of brain information processing ” from MEXT, Japan.

\section{REFERENCES}

[1] Muresan DD and Parks TW (2003), Adaptive principal components and image denoising. International Conference on Image Processing, 1, 101-4.

[2] Olshausen BA and Field DJ (1996), Emergence of simple-cell receptive field properties by learning a sparse code for natural images. Nature, 381(6583), 607-609.

[3] Elad M and Aharon M (2006), Image denoising via sparse and redundant representations over learned dictionaries. IEEE transactions on image processing, 15(12), 3736-45.

[4] Zhang L, Dong W, Zhang D, and Shi G (2010), Two-stage image denoising by principal component analysis with local pixel grouping, Pattern Recognition, 43, 1531-1549.

[5] Lee DD and Seung HS (1999), Learning the parts of objects by non-negative matrix factorization. Nature, 401(6755), 788-91.

[6] Kim YD and Choi S (2007), Nonnegative Tucker Decomposition. IEEE Conference on Computer Vision and Pattern Recognition, 1-8. 
[7] De Lathauwer L, De Moor B, and Vandewalle J (2000), On the Best Rank-1 and Rank$\left(R_{1}, R_{2}, \ldots, R_{N}\right)$ Approximation of Higher-Order Tensors. SIAM Journal on Matrix Analysis and Applications, 21(4), 1324-42.

[8] Bader BW and Kolda TG (2010), MATLAB Tensor Toolbox Version 2.4. 


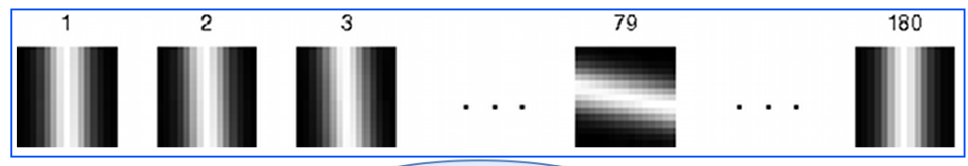

Inner product
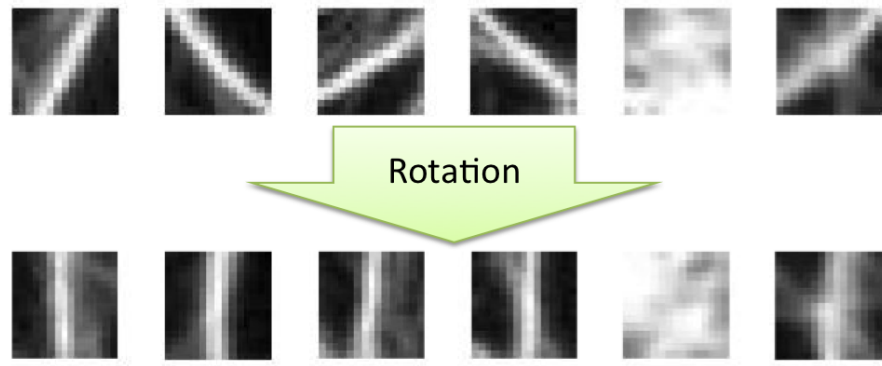

Fig. 1: Template image patch set by hand (leftmost panel) and its rotations $(2 \ldots 180)$. Foreground image patches before (middle) and after (lower) the rotation process.
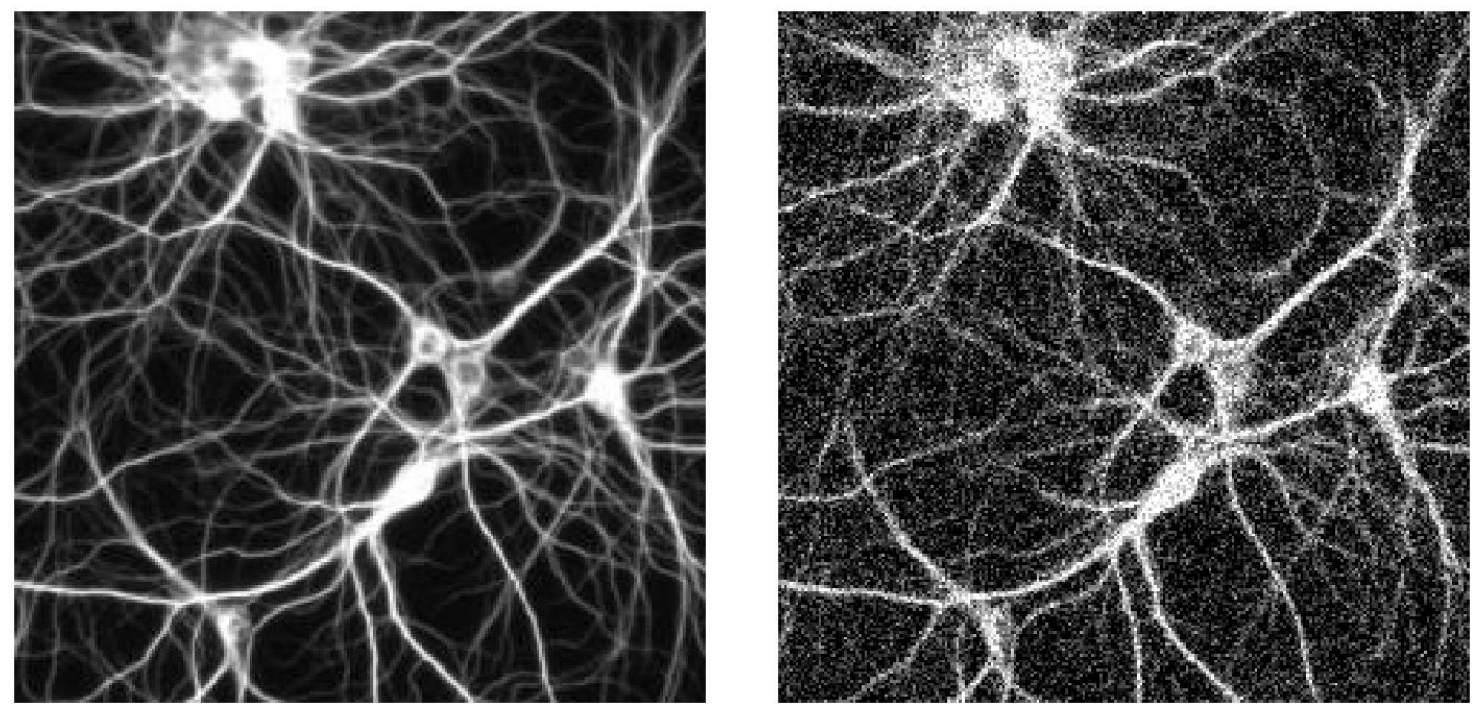

Fig. 2: An original microscopic image (size: $300 \times 300$ pixels) taken from www.greenspine.ca (Paul De Koninck, Laval University) (left) and its noisy image (right). 

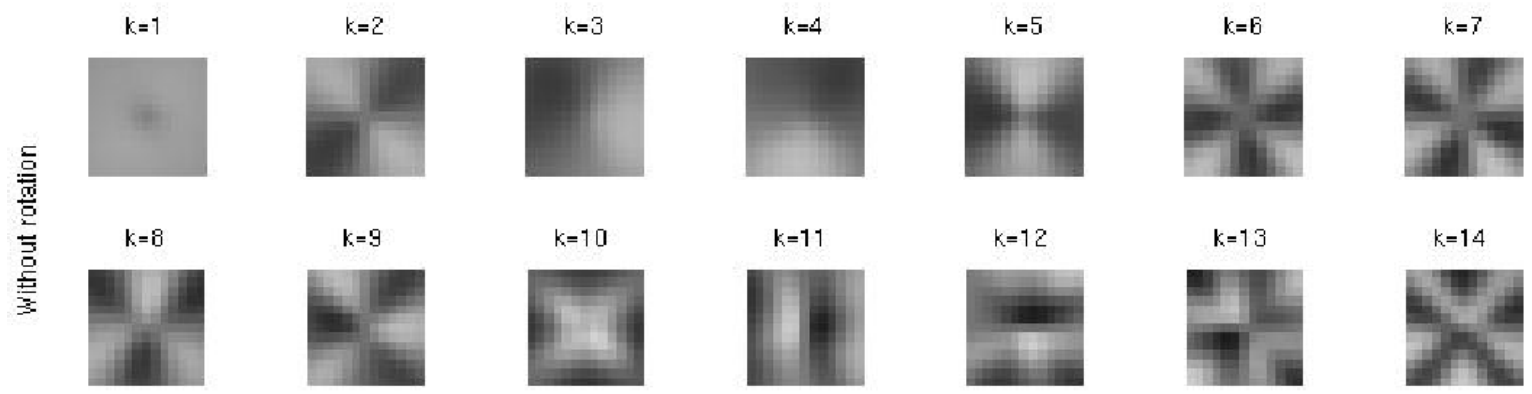

(a) matrix factorization without patch rotation
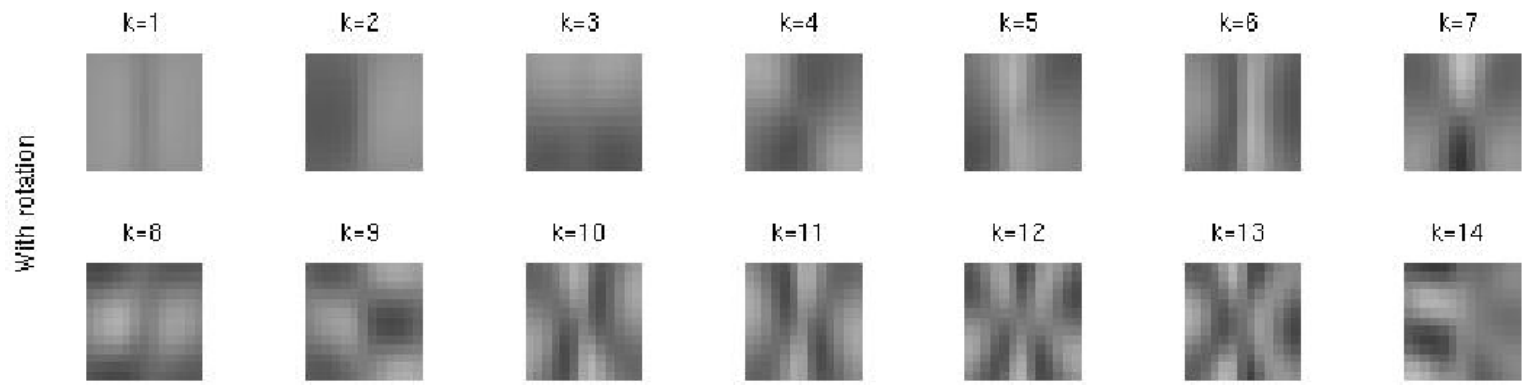

(b) matrix factorization with patch rotation
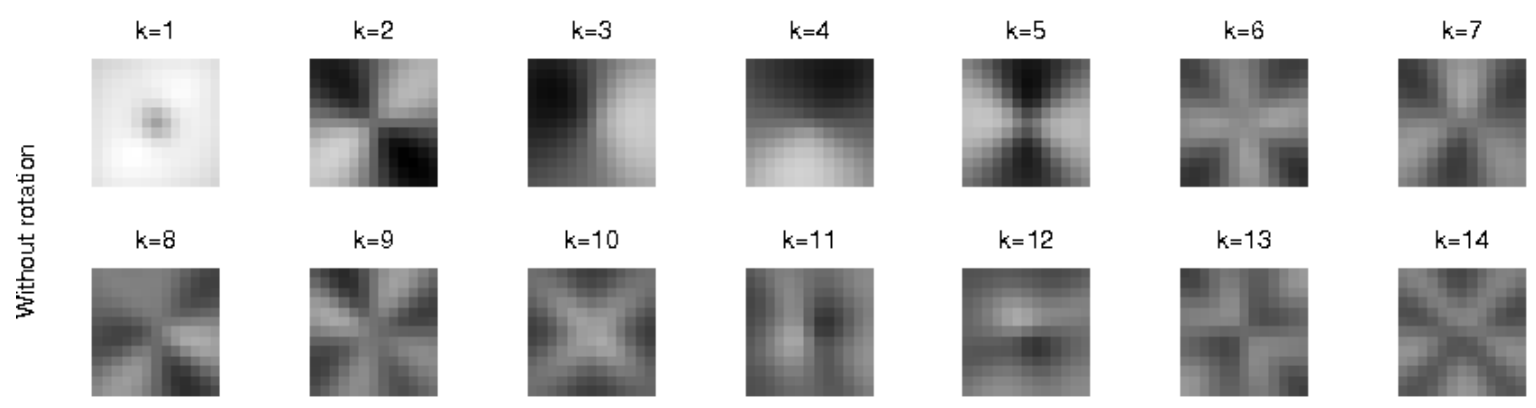

(c) tensor factorization without patch rotation
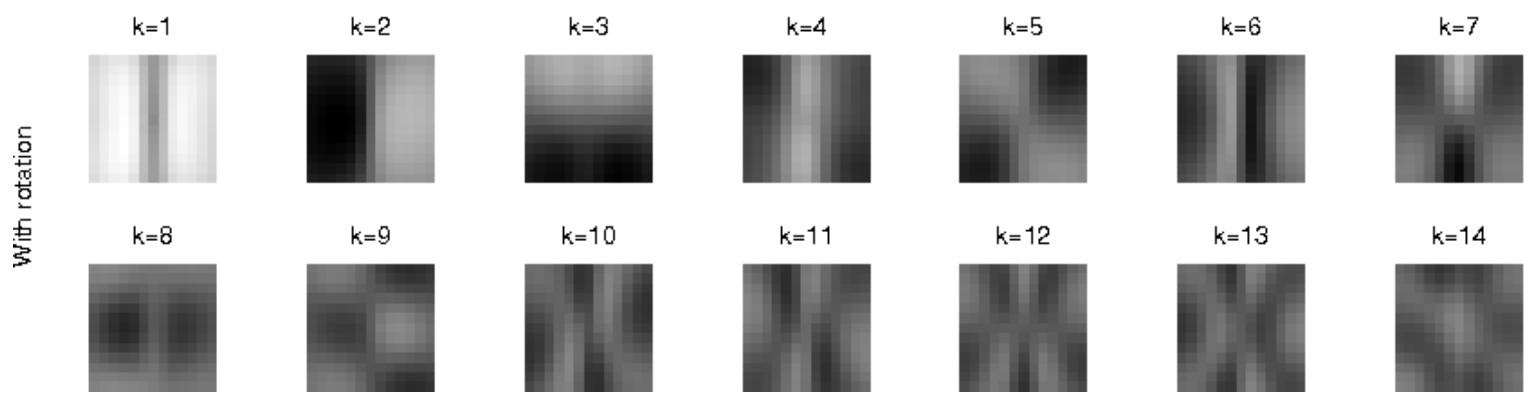

(d) tensor factorization with patch rotation

Fig. 3: Top 14 patch factor images obtained by each of four methods: (a) matrix factorization without patch rotation, (b) matrix factorization with patch rotation, (c) tensor factorization without patch rotation, and (d) tensor factorization with patch rotation. 


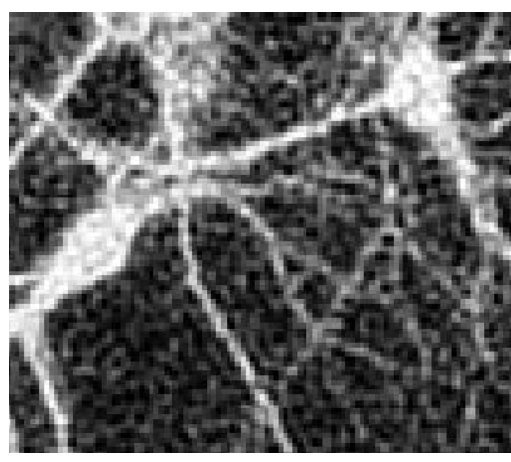

(a)

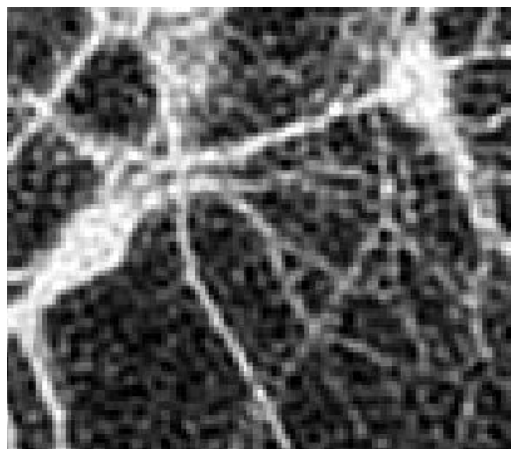

(c)

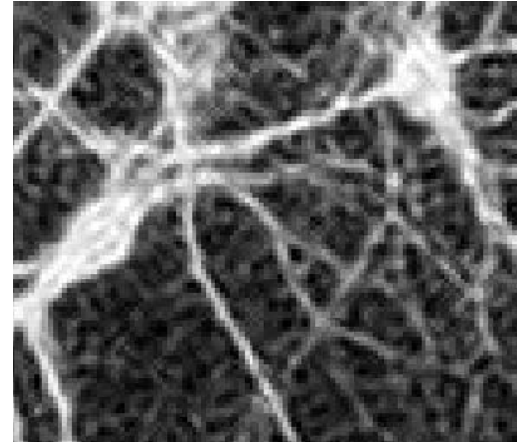

(b)

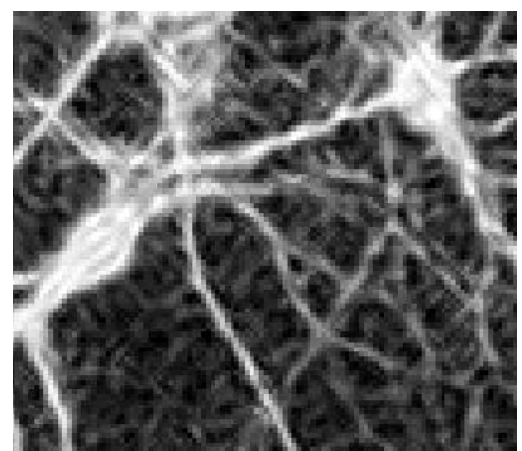

(d)

Fig. 4: Restoration by matrix factorization (a) without patch rotation $(K=64)$ and with patch rotation $(K=52)$. Restoration by tensor factorization (c) without patch rotation $(K=52)$ and (d) with patch rotation $(K=46)$.

Table 1: RMSE comparison for various noise levels added to the original image.

(a) variance $=0.01$

\begin{tabular}{|c|c|c|}
\hline RMSE & MF & TF \\
\hline without rotation & $18.27(K=70)$ & $17.48(K=64)$ \\
\hline with rotation & $17.28(K=64)$ & $\mathbf{1 6 . 4 2}(K=55)$ \\
\hline
\end{tabular}

(b) variance $=0.03$

\begin{tabular}{|c|c|c|}
\hline RMSE & MF & TF \\
\hline without rotation & $27.64(K=64)$ & $26.09(K=52)$ \\
\hline with rotation & $25.51(K=52)$ & $\mathbf{2 4 . 2 1}(K=46)$ \\
\hline
\end{tabular}

(c) variance $=0.1$

\begin{tabular}{|c|c|c|}
\hline RMSE & MF & TF \\
\hline without rotation & $44.26(K=55)$ & $42.11(K=49)$ \\
\hline with rotation & $41.01(K=46)$ & $\mathbf{3 8 . 9 9}(K=40)$ \\
\hline
\end{tabular}




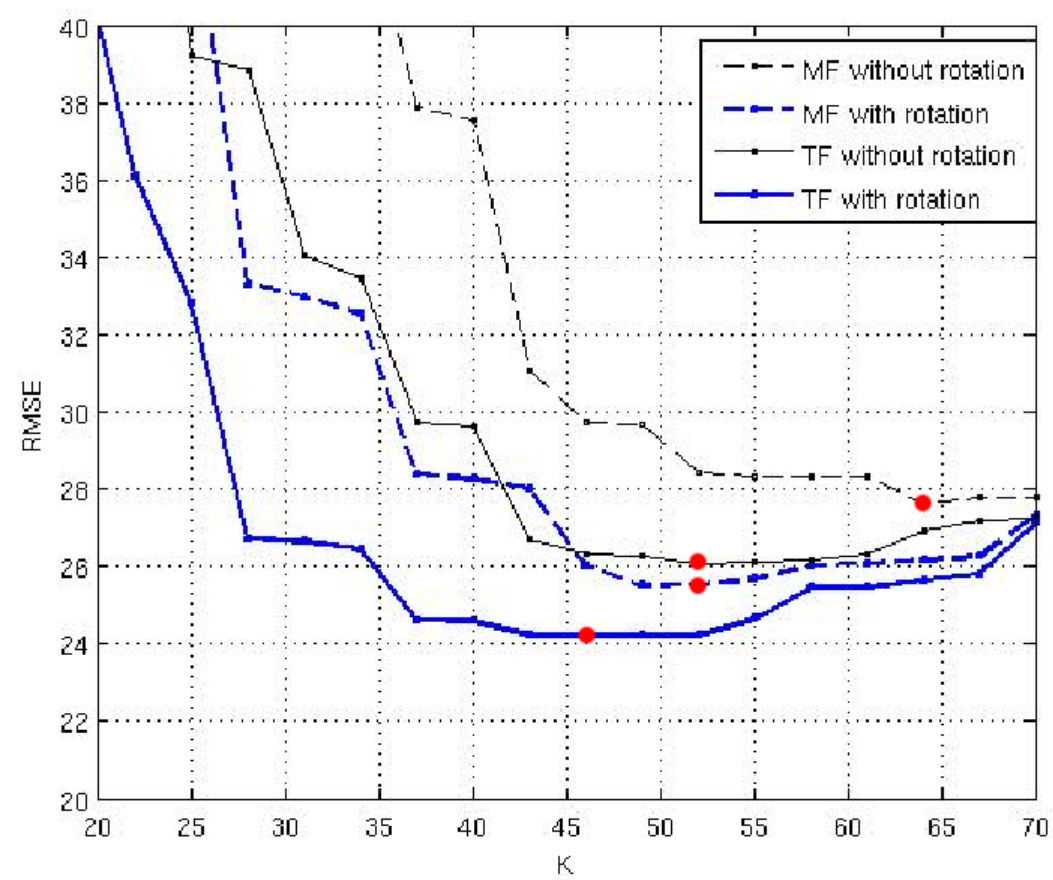

Fig. 5: Comparison of image restoration performance. Horizontal and vertical axes denote the number of bases $(K)$ and the root mean squared restoration error (RMSE), respectively. Solid and chained lines denote the image restoration with and without image patch rotation processes, respectively. Thick and thin lines denote the image restoration by means of three-way tensor factorization and matrix factorization, respectively. A red point denotes the best setting of $K$ for each case.

Input: $\underline{\mathbf{Y}} \in \mathbb{R}^{I_{1} \times I_{2} \times I_{3}}$

Output: $n$th matrix $\mathbf{A}^{(n)} \in \mathbb{R}^{I_{n} \times J_{n}}(n=1,2,3)$ and a core tensor $\underline{\mathbf{G}} \in \mathbb{R}^{J_{1} \times J_{2} \times J_{3}}$ such that $\|\underline{\mathbf{Y}}-\underline{\hat{\mathbf{Y}}}\|_{\mathrm{F}}^{2}$ is minimized.

1: begin

2: random initialization for all matrices $\mathbf{A}^{(n)}$

3: repeat

4: $\quad$ for $n=1$ to 3 do

5: $\quad \underline{\mathbf{Z}}^{(n)}=\underline{\mathbf{Y}} \times{ }_{-n}\left\{\mathbf{A}^{(n)^{\top}}\right\}$

6: $\quad\left[\mathbf{A}^{(n)}, \boldsymbol{\Sigma}^{(n)}, \mathbf{B}^{(n)}\right]=\operatorname{svds}\left(\mathbf{Z}_{(n)}^{(n)}, J_{n}\right)$

7: $\quad$ end for

8: until a stopping criterion is realized

9: $\underline{\mathbf{G}}=\underline{\mathbf{Y}} \times{ }_{1} \mathbf{A}^{(1)^{\top}} \times{ }_{2} \mathbf{A}^{(2)^{\top}} \times{ }_{3} \mathbf{A}^{(3)^{\top}}$

10: end

Fig. 6: A pseudo code of HOOI algorithm for three-way tensor factorization 